its derivatives. Accumulation of propionate derivatives in the cytoplasm and plasma leads to intra- and extracellular metabolic acidosis. The clinical manifestation is severe; arrhythmia and cardiomyopathy (hypertrophic and dilated) are cited as major causes of death. Little is known of the underlying mechanisms. Since butyrate is in the same homologous series as propionate, and is itself a well-established inhibitor of histone deacetylases (HDACs), it is plausible pro-hypertrophic signalling in PA is epigenetically regulated. Therefore, we investigated the effects of propionate on cardiomyocytes.

In preliminary work, culturing neonatal rat ventricular myocytes (NRVMs) in a chemical milieu mimicking PA (6 mM propionate; $200 \mathrm{M}$ ammonium; $18 \mathrm{mM}$ bicarbonate; $\mathrm{pH}$ 7.15) increased significantly cardiomyocyte area. Treating adult rat VMs with PA-medium increased significantly histone-3 lysine-9 acetylation, phenocopying the effects of butyrate. To determine the gene expression profile associated with PA, RNA-sequencing was performed on polyadenylated RNA extracted from NRVMs, treated with control-, butyrate-, or PA-medium. In PA-medium, Differentially Expressed Gene analysis demonstrated $3.0 \%$ of genes were upregulated and $3.0 \%$ downregulated (FDR $<0.0001)$. Gene Ontology Enrichment Analysis using DAVID revealed 'cardiac muscle contraction' and 'metabolic pathways' as being significantly enriched. $1.9 \%$ of genes were upregulated in both PA and butyrate, representing candidates that could be regulated by HDACs. We are currently validating these hits.

\section{P9 HYPERPOLARISED KETONE BODY METABOLISM IN THE RAT HEART}

1,2JJ Miller, ${ }^{1,3}$ YB Ding, ${ }^{1} \mathrm{D}$ Ball, ${ }^{1,4} \mathrm{AZ}$ Lau, ${ }^{1} \mathrm{DJ}$ Tyler. ${ }^{1}$ Department of physiology, Anatomy and Genetics, Sherrington Building, University of Oxford, Oxford; ${ }^{2}$ Department of Physics, Clarendon Laboratory, University of Oxford, Oxford; ${ }^{3}$ Department of Chemistry, Dyson Perrins, South Parks Road, Oxford; ${ }^{4}$ Health Sciences, Sunnybrook Research Institute, Toronto, Canada

\subsection{6/heartjnl-2018-BSCR.14}

Hyperpolarised Magnetic Resonance Spectroscopy (MRS) permits the real time determination of metabolic fluxes in the living heart. In contrast to conventional thermal-equilibrium MRS, the hyperpolarisation technique increases the signal-tonoise ratio of acquired spectra by many orders of magnitude, and therefore allows isotopically labelled probes to be injected into an organism and followed through their subsequent biochemical pathways.

We show here that $[1-13 C]$ acetoacetate and $[1-13 C] \beta$ hydroxybutyrate can be hyperpolarised and probe ketone body metabolism in both the ex vivo perfused and in vivo rat heart. Downstream metabolites were observed within the perfused heart, including acetylcarnitine, citrate, and glutamate. In the in vivo heart, a statistically significant increase in acetylcarnitine production from acetoacetate was observed in the fed state, as well as a potential reduction in glutamate, when compared to fasted controls.

The metabolism of acetoacetate and $\beta$-hydroxybutyrate is known to be altered in various disease states, including diabetic cardiomyopathy, and this proof-of-principle study shows that hyperpolarisation can probe the role of ketone bodies in the diseased heart. The increased rate of acetylcarnitine production following feeding is consistent with its reported role as a store of acetyl moieties should they be abundant in a post-prandial state, into which ketone oxidation is directed. In the fasted state, apparent glutamate levels were higher, which is consistent with an increased flux of ketone bodies into the TCA cycle during fasting.

Further work will aim to quantify these fluxes, and explore the role of ketone bodies in animal models of cardiac disease, such as diabetic cardiomyopathy.

\section{P10 MIR-133A OVEREXPRESSION IMPAIRS ENDOTHELIAL CELL MIGRATION AND TUBE FORMATION IN VITRO}

${ }^{1}$ Sathishkumar Kurusamy, ${ }^{1}$ Jude C Ihugba, ${ }^{1}$ Angel L Armesilla*, ${ }^{2}$ James M Cotton*. ${ }^{1}$ Cardiovascular Molecular Pharmacology Laboratory, School of Pharmacy, Research Institute in Healthcare Science, Faculty of Science and Engineering, University of Wolverhampton, Wolverhampton, UK; ${ }^{2}$ Department of Cardiology, Heart and Lung Centre, New Cross Hospital, Wolverhampton, UK; *Equal senior authors

\subsection{6/heartjnl-2018-BSCR.15}

Emerging evidence indicates that short ( 22 nucleotides) noncoding RNA molecules called microRNAs (miRNAs) play a key role in the regulation of post-ischaemic angiogenesis. miRNAs control gene expression by binding to complementary sequences of specific mRNA transcripts, leading to degradation or translational repression of the targeted mRNA. miR-133a is highly expressed and plays a crucial role in skeletal and cardiac muscle biology. The expression level of miR-133a in vascular endothelial cells is very low under physiological conditions. However, multiple cardiovascular risk factors including oxidized-LDL, pro-inflammatory cytokines, hyperglycemia, dyslipidemia, and hyperhomocysteinemia induce aberrant miR-133a expression in endothelial cells leading to endothelial dysfunction. Endothelial dysfunction in cardiovascular ischaemic disease is often accompanied by impairment of reparative angiogenic processes.

Here, we have evaluated the role of aberrant miR133a expression in VEGF-mediated angiogenesis. We show that exogenous expression of miR-133a in Human Umbilical Vein Endothelial Cells (HUVEC) significantly reduces VEGF-induced endothelial cell proliferation. Moreover, adenoviral-mediated delivery of cardiac miR133a into endothelial cells inhibits HUVEC cell motility and tubular morphogenesis. Interestingly, downregulation of phosphorylation (activation) of the signalling protein Erk1/2 by miR-133a was detected in VEGF stimulated HUVEC cells.

Our results demonstrate that aberrant expression of miR133a has an anti-angiogenic effect in endothelial cells, and strongly suggest that targeted strategies to suppress ectopic expression of miR-133a in the dysfunctional endothelium might have important therapeutic applications to improve reparative angiogenic process in patients suffering from ischaemic cardiovascular disease.

Acknowledgement This work was supported by the Rotha Abraham Bequest Charity 